\title{
Low Power Photovoltaic Inverters Built up with SiC JFETs
}

\author{
Balázs Farkas*, Ernő Paál*, Károly Veszprémi** \\ *Hyundai Technologies Center Hungary \\ Hermina út 22, H-1146 Budapest, Hungary, bfarkas@h-tec.hu, epaal@h-tec.hu \\ **Department of Electric Power Engineering, Budapest University of Technology \\ and Economics \\ Egry J. u. 18, V1, H-1111 Budapest, Hungary, veszpremi.karoly@ vet.bme.hu
}

\begin{abstract}
The new generation of semiconductor switching devices based on silicon carbide makes higher efficiency and power density possible in photovoltaic $(P V)$ inverters among many applications. The new type of JFET requires new driver circuits and a more careful arrangement of them. The main circuit arrangement has to have low stray inductance to ensure low overvoltage spikes and switching losses. It is well known that converter efficiency is an important parameter in the PV industry. SiC devices offer a good possibility to achieve 99\% efficiency. This paper approaches the topic from the application point of view.
\end{abstract}

\section{Introduction}

As a result of recent researches and developments, new switching devices have become available on the market. Among the appeared products, SiC normally on and normally off JFETs have favorable attributions [1]. Additionally, it is well known that switching devices like MOSFETs or IGBTs have conduction and switching losses. Power semiconductors usually work in hard switching operation mode in PV inverters. There are some basic requirements to provide optimal operation of JFETs. Driver circuits of the JFET have a crucial role to ensure proper operation during conduction state and during turn on and turn off [2]. The high frequency operation with low switching losses enables high power density in converters.

Basically, there are two types of JFETs: normally off and normally on. Normally off JFETs are in turned off state when gate-source voltage is below its threshold voltage. While, normally on JFETs are in turned on state when gate-source voltage is below its threshold voltage. 


\section{Silicon Carbide Power JFET Features}

Normally on type:

- Positive temperature coefficient, it makes the parallel connection easier

- Extremely fast switching with no "tail" current

- High maximum operating temperature

- Low, but temperature sensitive $\mathrm{R}_{\mathrm{DSonmax}}$

- Temperature sensitivity of the $\mathrm{R}_{\mathrm{DSon}}$ is smaller than Si MOSFETs

- Voltage controlled

- Low gate charge

- Low intrinsic capacitance

- High short circuit withstand capability

- Bidirectional current conduction

- Unipolar device

Normally off type:

- Compatible with standard gate driver ICs

- Positive temperature coefficient, it makes the parallel connection easier

- Temperature Independent Switching Behavior

- High maximum operating temperature

- Low, but temperature sensitive $\mathrm{R}_{\mathrm{DSonmax}}$

- Temperature sensitivity of the $\mathrm{R}_{\mathrm{DSon}}$ is smaller than Si MOSFETs

- Voltage controlled but low $\mathrm{R}_{\mathrm{DSon}}$ requires permanent gate current

- Low gate charge

- Low intrinsic capacitance

- High short circuit withstand capability

- Extremely fast switching with no "tail" current

- Bidirectional current conduction

- Unipolar device 


\section{Gate Driver for Normally Off Type}

\subsection{Steady-State Operation}

In the driving of JFET not only gate-source voltage but also gate bias current are important in order to ensure device saturation. On the other hand, it is important to avoid unnecessary extra losses on the parasitic gate source diode.

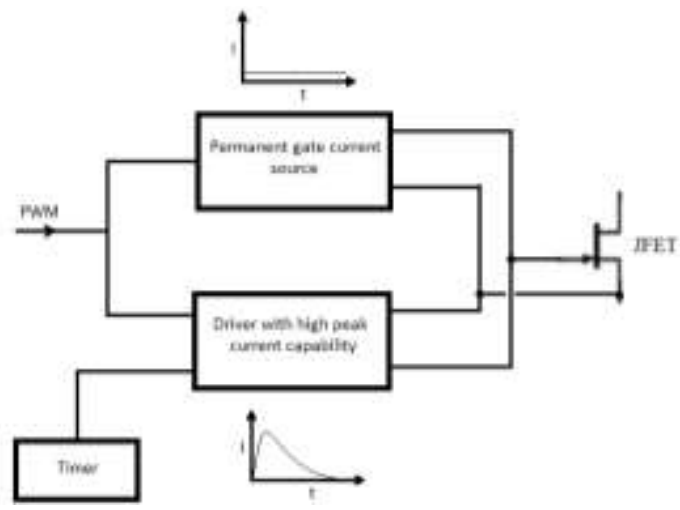

Figure 1

Typical gate driver circuit for Normally off JFET

\subsection{Turn On}

A high peak value and low rise time of the gate forward current are necessary to reduce turn on losses (see Fig. 1). Inductance of the gate-source current loop is crucial so as to achieve low rise time and oscillation free switching. Therefore, it is practical to reduce the distance between JFETs terminal and gate boost unit. Apart from the distance, induction free layout is critical as well.

Commutation from $\mathrm{SiC}$ freewheeling diode during the turn on of JFET can cause high frequency oscillation in the drain current. The main reason of this oscillation is the charge of the parasitic capacitances and inductances. Since the output capacitance of the JFET is very low, the main source of these capacitances is the PCB structure and diode.

The application of ferrite bead in the gate makes it possible to reduce the current oscillation in the gate loop. However, it increases turn on time and turn on losses.

The steady state value of the gate voltage is limited to $3 \mathrm{~V}$ by the parasitic gate source diode. As a consequence of this, the difference between turn on level of the gate voltage and threshold voltage is relatively small. 


\subsection{Turn Off}

Turn off delay and fall time can be reduced by negative turn off voltage. Negative turn off voltage can prevent parasitic turn on. An inappropriate suppression of parasitic turn on can cause additional power losses in the phase legs. The use of an additional capacitor (1-5 nF) parallel with the gate-source terminals can suppress this phenomenon.

PCB stray capacitances must be small; otherwise voltage rise time during turn off transient can be dominated by them. Therefore, the PCB layout design is a critical point of SiC JFET application. These capacitances reduce overvoltage and turn off losses, but increases turn on losses and current oscillation during turn on.

\subsection{Layout of Gate Driver}

Distance between gate boost unit and stray inductances have to be minimized in order to achieve fast and oscillation free switching.

The power supply decoupling capacitors have to be also located very close to the driver boost unit. The gate resistor of the driver should be a non-inductive type.

Due to the high switching speed of SiC JFET, switching noises can propagate across the supply system. Therefore, it is essential to use a high inductive component with high bandwidth or transformers with low couple capacitances in order to attenuate this noise.

\section{JFET Application in H-Bridge and Boost Converter of PV Inverters}

A traditional low-power inverter with two or more levels can be constructed with MOSFETs or IGBTs and it usually operates with approx. $16 \mathrm{kHz}$ switching frequency to provide proper reduction of audible noise. Because of the efficiency, switching losses have to be kept at an admissible level.

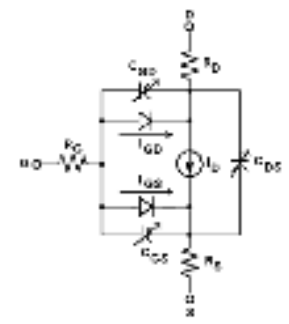

Figure 2

Typical gate driver circuit for Normally off JFET 
A switching frequency of $100-150 \mathrm{kHz}$ is achievable thanks to the high switching speed of SiC JFET [3], [6]. It is important to take into consideration the impact of higher operation frequency on other components. On the one hand, grid side filter including reactors and capacitors and booster choke can be significantly reduced, but on the other hand, total loss of the system is increased, due to switching losses of JFET and additional losses in reactors and capacitors.

A 3-level $\mathrm{H}$-bridge with power of $3 \mathrm{~kW}$ was constructed at the laboratory of Hyundai Technologies Center to investigate JFET operation. In that system the switching frequency of single-phase $\mathrm{H}$-bridge is around $32 \mathrm{kHz}$, which is twice as high as the conventional value at Fig. 3. The boost circuit frequency is around 60 $\mathrm{kHz}$.

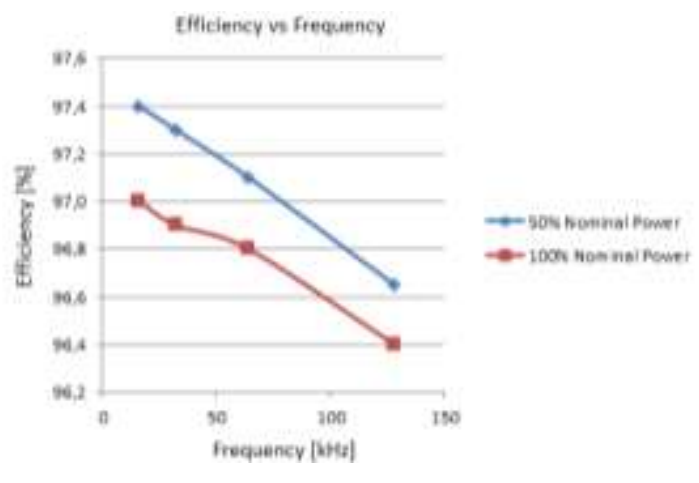

Figure 3

Comparison of $\mathrm{H}$-bridge efficiencies at switching frequencies

Higher frequency operation and more output level of $\mathrm{H}$-bridge make the reduction of inductive component in grid side filter possible. The required inductivity is around $35 \%$ compared to the conventional $\mathrm{H}$ bridge inverter.

Thanks to switching frequency of $60 \mathrm{kHz}$, the required booster choke is less than $50 \%$ compared to $16 \mathrm{kHz}$ operation.

Not only normally-off, but also normally-on JFETs in the cascade circuit were tested.

\subsection{Normally on JFET Application}

This kind of JFET is turned on when gate source voltage is below the threshold voltage. As a result of this, normally on type JFETs are not allowed to be used in H-Bridge without any measures. Auxiliary MOSFET should be used in order to avoid the short-circuit of the DC link.

Fig. 4 shows the cascade configuration. In this configuration, the JFET gating energy is provided by the main circuit. This means that only the low voltage 
MOSFET requires energy from an auxiliary power supply. The surge current capability of cascade configuration is deteriorated, because the short-circuit withstand time of normally on JFETs is much higher than low voltage MOSFET. Due to this fact, the short-circuit at DC link by half bridge leg destroys first the MOSFET and then the JFET very fast. As a consequence, the protection system has very short time to handle the failure, so it is difficult to design short circuit protection similar to IGBTs.

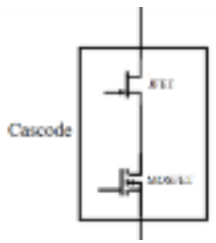

Figure 4

Cascade arrangement of a switching device

Cascade structure is very sensitive to parasitic inductance MOSFET-JFET gate loop. It can cause oscillation during the switching, which increases switching losses. Due to the fact that the aforementioned PCB stray capacitances are parallel with SiC JFET, they make SiC JFET slower and therefore, the voltage of MOSFET can be dangerously high.

There is a possibility to drive JFET and MOSFET separately [4], [5]. Before the voltage level at power supply reaches the nominal range, MOSFET and JFET are in off state. When the auxiliary power supply is high enough, MOSFET is turned on continuously and only JFET is controlled. This solution provides a better switching behavior but requires a higher gating power supply energy.

\subsection{Normally Off JFET Application}

Drivers of JFETs need energy from the auxiliary power supply to turn on the devices, but without the auxiliary power supply, the semiconductors are in turned off state. JFETs have robust short circuit withstand capability, therefore it is easy to equip them with overcurrent protection.

\section{Test Results of JFET Applications in PV Inverter}

The tests were carried out on an experimental $3 \mathrm{~kW}$ single-phase, transformerless $\mathrm{H}$-bridge inverter. 


\subsection{Main Technical Data}

Rated power:

$3 \mathrm{~kW}$

Max. DC input voltage:

$550 \mathrm{~V} \mathrm{dc}$

Input current max.:

$17 \mathrm{~A}$

MPP range:

$150-450 \mathrm{~V}$

Output current:

$16 \mathrm{~A}_{\mathrm{RMS}}$

Output voltage:

$230 \mathrm{~V}_{\mathrm{RMS}}$

Output frequency:

$50 / 60 \mathrm{~Hz}$

$\cos (\varphi)$ :

0.8 -1 leading or lagging

Applied instruments:

Oscilloscope:

type Yokogawa DLM $6054 / 500 \mathrm{MHz}$

Voltage probes:

type Yokogawa DLM 701939 / 500 MHz

Voltage probes:

type Yokogawa DLM 701945 / 500 MHz

Voltage probes:

type PBA 1000 Yokogawa $701912 / 1 \mathrm{GHz}$

Current probe:

type DLM $701933 / 50 \mathrm{MHz}$

\subsection{Boost Unit Operation}

Fig. 5 shows the switching behavior of JFET with switching frequency of approximately $60 \mathrm{kHz}$. There is a current spike during turn on caused by the booster $\mathrm{SiC}$ diode capacitive charge. Current spike can also be reduced by the application of a ferrite bead in the gate circuit. Results are shown in Fig. 6. This is also effective against gate ringing current, however its consequences are slower switching speed and additional switching losses.

Fig. 5a shows a turn on and a turn off transition at app. 50\% duty cycle with boosted voltage of $550 \mathrm{~V}_{\mathrm{DC}}$ and load current of $13 \mathrm{~A}$. In Fig. $5 \mathrm{~b}$ and $5 \mathrm{c}$ an enlarged single period of turn on and turn off can be seen. In this case the gate does not contain a ferrite bead. 


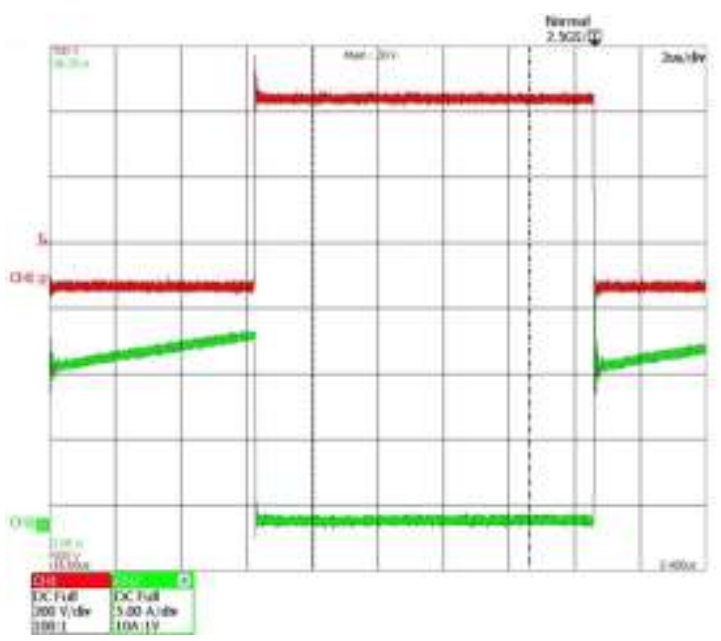

Figure 5a

CH1 Cascade voltage, $\mathrm{CH} 2$ Cascade Drain current

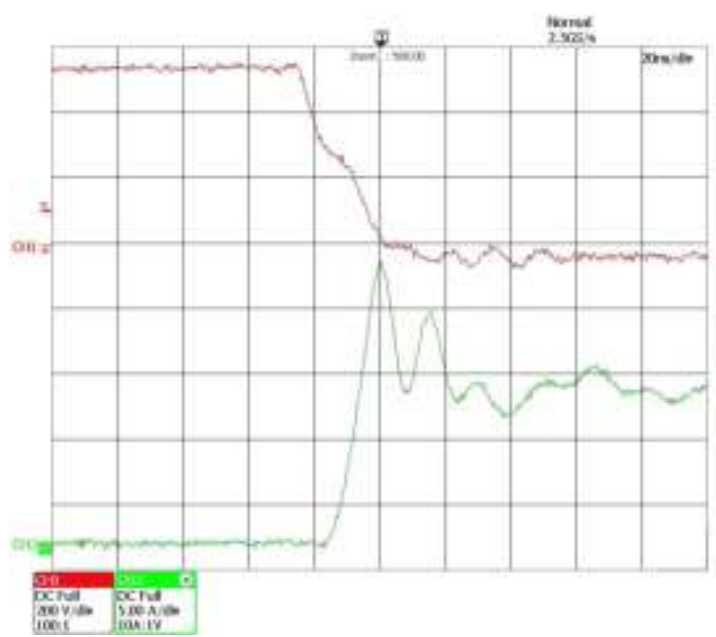

Figure 5b

CH1 Cascade voltage, $\mathrm{CH} 2$ Cascade Drain current 


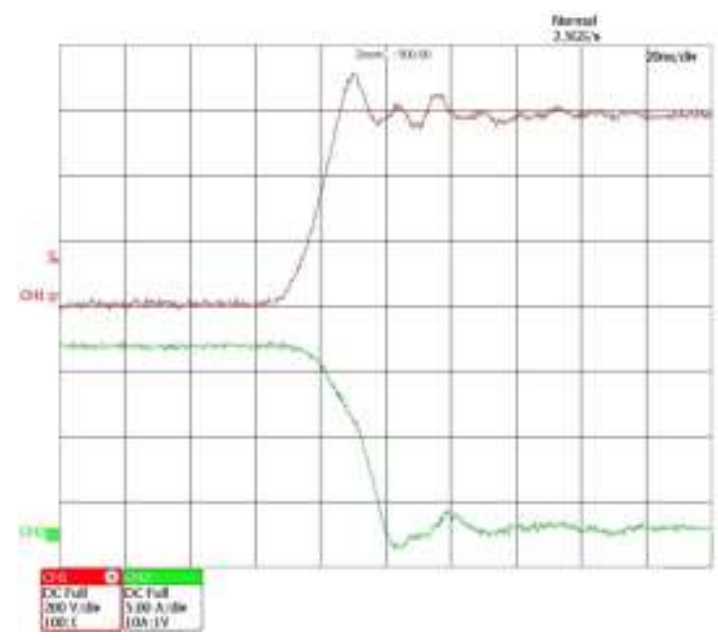

Figure 5c

CH1Cascade voltage, $\mathrm{CH} 2$ Cascade Drain current

Fig. 6 shows similar operation conditions that Fig. 5, but the gate contains a ferrite bead.

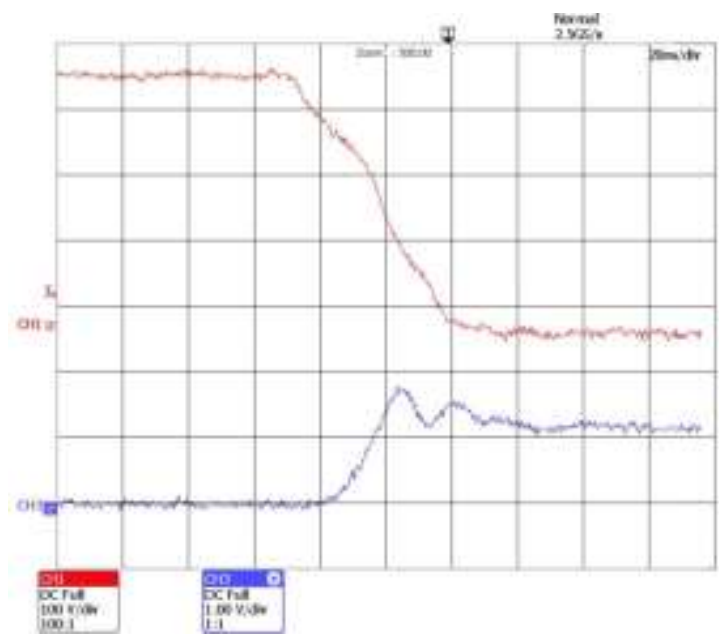

Figure 6a

CH1 Cascade voltage, $\mathrm{CH} 2$ Cascade Drain current (100 mV/A) 


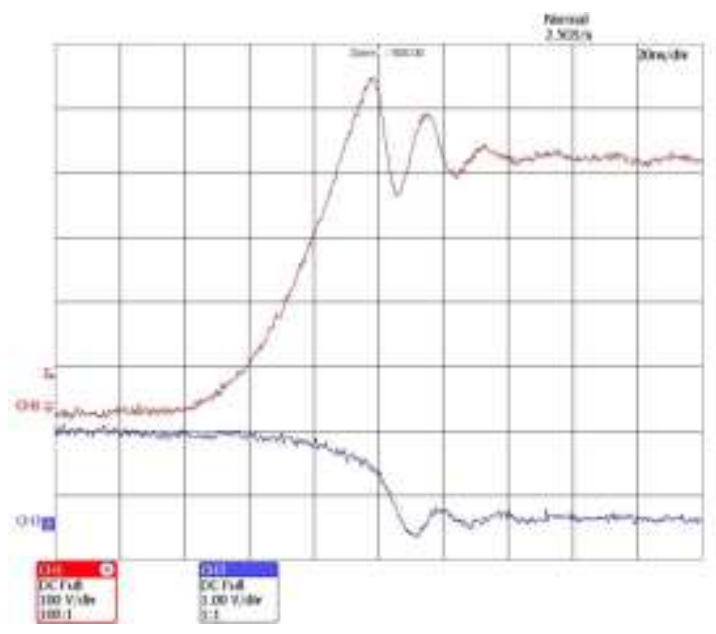

Figure $6 b$

CH1 Cascade voltage, CH3 Cascade Drain current (100mV/A)

\subsection{H-Bridge Unit Operation}

There is a dangerous effect in the phase legs during on state of the top JFET device. The bottom JFET can have a parasitic turn on due to the miller effect.

It is advisable to apply a negative gate-source voltage to keep the JFET in the turned off state. Aforementioned sensitivity can be further decreased by means of parallel connected gate capacitor of few $\mathrm{nF}$. The experimental $\mathrm{H}$-bridge was not sensitive to those voltage stresses. Fig. 7 shows current and voltage of the top semiconductor.

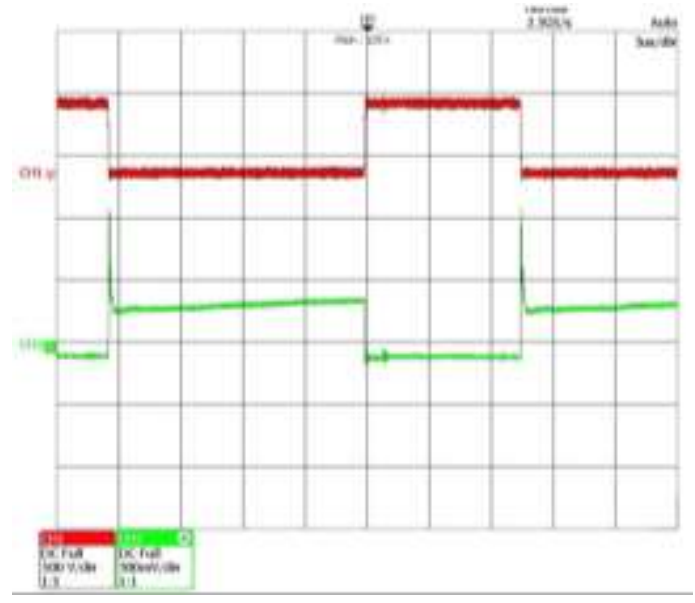

Figure 7

CH1Cascade voltage, CH2 Cascade Drain current (20 mV/A) 
A half sinusoidal output current of the inverter is recorded in Fig. 8, where the switching frequency was $30 \mathrm{kHz}$. In the same figure the output modulated voltage was recorded in blue. Enlarged signals can be seen at the bottom of Fig. $8 \mathrm{~b}$.

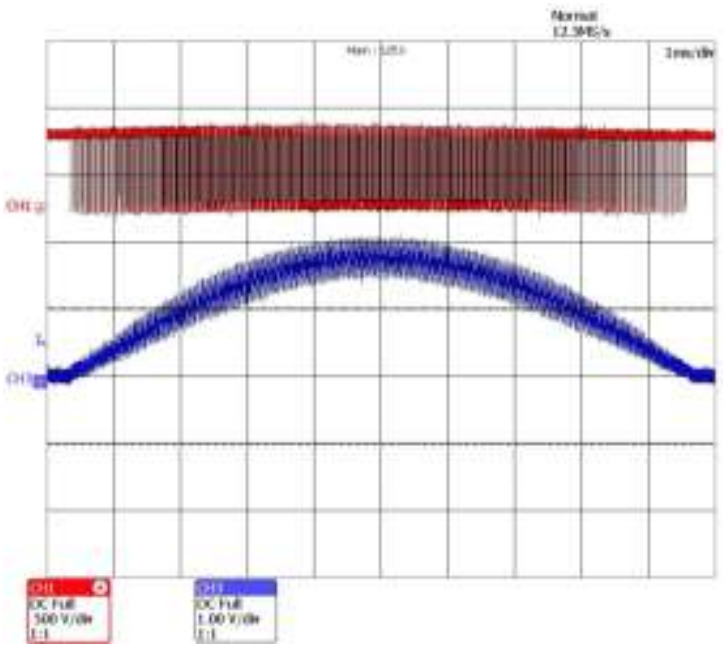

Figure 8a

Output current and output voltage of the inverter

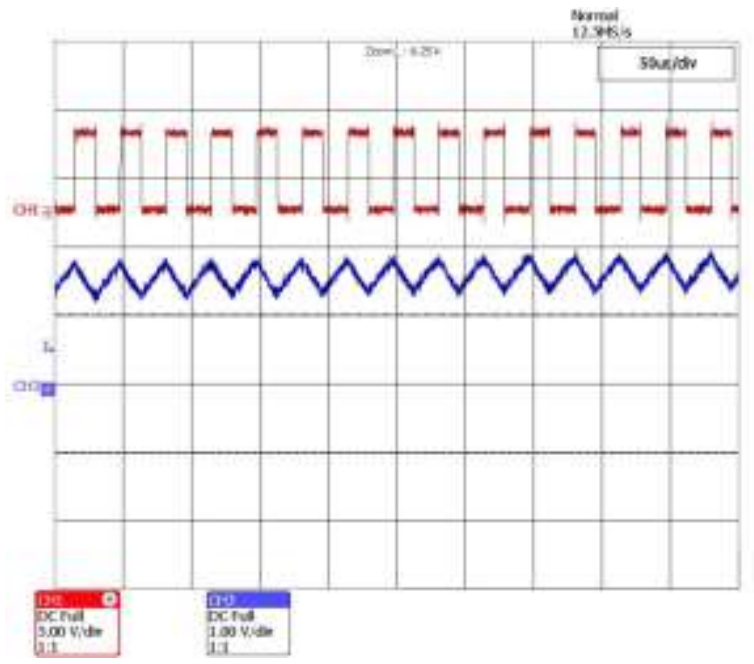

Figure $8 b$

Half sinusoidal output current $500 \mathrm{~V}_{\mathrm{DC}}(500 \mathrm{~V} / \mathrm{DIV})$ voltage and $20 \mathrm{~A}(13.33 \mathrm{~A} / \mathrm{DIV})$ load current, modulation frequency $30 \mathrm{kHz}$ 


\section{Conclusions}

This paper focuses on the application of a new semiconductor class in low power PV inverters. In addition it introduces various solutions and some key rules for circuit design and construction.

The paper points out the advantages and disadvantages of normally on and off JFETs. Furthermore, it presents the measurement results of an experimental PV inverter.

\section{Acknowledgement}

This work was supported by Hyundai Technologies Center Hungary Ltd.

\section{References}

[1] A. Ritenout, V. Bondarenko, R. Kelley and D. C. Sheridan: Electrical Characterization of Large Area 800V Enhancement- Mode SiC VJFET for High Temperature, Material Science Forum, March 2009, pp. 715-718

[2] R. Kelley and M. S. Mazzola: SiC JFET Gate Driver Design for Use in DC/DC, APEC '06, March 2006, pp. 179-182

[3] B. Burger, B. Goeldi, Dirk Kranzer and Heribert Scmidt: 98.5\% Inverter Efficiency with SiC MOSFETs in $23^{\text {rd }}$ EU PVSEC, Valencia, Spain, $1^{\text {st }}$ to $4^{\text {th }}$ September 2008

[4] D. Domes, X. Zhang: Cascade Light Normally on JFET Standalone Performance in Normally off Cascade Ciruit. PCIM Europe, 2010

[5] K. Mino, S. Herold and J. W. Kolar: A Gate Drive Circuit for Silicon Carbide JFET, IECON '03, Nov. 2003, pp. 1162-1166

[6] H. Zhang, L. M. Tolbert: Efficiency of SiC JFET-based Inverters, $4^{\text {th }}$ ICIEA, May 2009, pp. 2056-2059 